\title{
The Relationship between Preoperative Wound Classification and Postoperative Infection: A Multi-Institutional Analysis of 15,289 Patients
}

\author{
Lauren M Mioton ${ }^{1}$, Sumanas W Jordan ${ }^{2}$, Philip J Hanwright ${ }^{2}$, Karl Y Bilimoria ${ }^{3}$, John YS Kim² \\ ${ }^{1}$ Department of Plastic Surgery, Vanderbilt School of Medicine, Nashville, TN; ${ }^{2}$ Division of Plastic and Reconstructive Surgery, ${ }^{3}$ Department \\ of Surgery, Northwestern University, Feinberg School of Medicine, Chicago, IL, USA
}

Background Despite advances in surgical techniques, sterile protocols, and perioperative antibiotic regimens, surgical site infections (SSIs) remain a significant problem. We investigated the relationship between wound classification (i.e., clean, clean/contaminated, contaminated, dirty) and SSI rates in plastic surgery.

Methods We performed a retrospective review of a multi-institutional, surgical outcomes database for all patients undergoing plastic surgery procedures from 2006-2010. Patient demographics, wound classification, and 30-day outcomes were recorded and analyzed by multivariate logistic regression.

Results A total of 15,289 plastic surgery cases were analyzed. The overall SSI rate was 3.00\%, with superficial SSIs occurring at comparable rates across wound classes. There were similar rates of deep SSIs in the clean and clean/contaminated groups $(0.64 \%)$, while rates reached over 2\% in contaminated and dirty cases. Organ/space SSIs occurred in less than 1\% of each wound classification. Contaminated and dirty cases were at an increased risk for deep SSIs (odds ratios, 2.81 and 2.74, respectively); however, wound classification did not appear to be a significant predictor of superficial or organ/space SSIs. Clean/contaminated, contaminated, and dirty cases were at increased risk for a postoperative complication, and contaminated and dirty cases also had higher odds of reoperation and 30-day mortality.

Conclusions Analyzing a multi-center database, we found that wound classification was a significant predictor of overall complications, reoperation, and mortality, but not an adequate predictor of surgical site infections. When comparing infections for a given wound classification, plastic surgery had lower overall rates than the surgical population at large.

Keywords Plastic surgery / Postoperative complications / Wound infection
Correspondence: John YS Kim

Division of Plastic and Reconstructive Surgery, Northwestern University, Feinberg School of Medicine, 675 North St. Clair Street, Galter Suite 19-250, Chicago, IL 60611, USA Tel: +1-312-695-6022

Fax: +1-312-695-5672

E-mail: jokim@nmh.org

Received: 8 Jan 2013 • Revised: 11 Apr 2013 • Accepted: 7 May 2013

pISSN: 2234-6163 • elSSN: 2234-6171 • http://dx.doi.org/10.5999/aps.2013.40.5.522・ Arch Plast Surg 2013;40:522-529

Lauren M. Mioton is funded on a research scholarship through Vanderbilt University School of Medicine by NIH CTSA Grant UL1RR024975.

No potential conflict of interest relevant to this article was reported.

\section{INTRODUCTION}

While strides have been made to improve the safety of surgical practices and decrease postoperative complications in the US, surgical site infections (SSIs) remain a significant obstacle in health care. Specifically, surgical site infections are the second most common hospital acquired infection (HAI) and the second most frequent adverse event experienced by a hospitalized pa- 
tient in the US. The Centers for Disease Control and Prevention (CDC) reports that two to five out of every 100 patients undergoing surgery will incur a postoperative infection. Patients who suffer from a surgical site infection are more likely to be readmitted within 30 days and carry a two-fold increased mortality risk $[1,2]$. Several analyses have shown that SSIs are independently associated with increased length of stay and costs [3-6]. Moreover, wound infections are often associated with unfavorable scarring, both aesthetic and functional, and in oncologic reconstructive cases, may delay adjuvant therapies.

\section{Historical background}

Several factors have been shown to influence surgical wound healing and postoperative infection rates. In 1964, the National Academy of Sciences introduced a surgical wound classification system based on the degree of microbial contamination to aid in infectious risk assessment, perioperative protocol development, and surgical decision-making. The classification system categorized all surgeries into one of four groups: clean, clean/contaminated, contaminated, and dirty (Table 1) [7]. Shortly thereafter, the National Nosocomial Infections Surveillance (NNIS) System was established by the $\mathrm{CDC}$ to monitor reported nosocomial infections in hospitals across the United States. In 1985, the CDC released guidelines for reducing postoperative wound infections, including recommendations for prophylactic antibiotics and improved sterile protocol. This report also estimated surgical site infection rates stratified by the four wound classifications: $1 \%$ to $5 \%$ for clean, $3 \%$ to $11 \%$ for clean/contaminated, $10 \%$ to $17 \%$ for contaminated, and over $27 \%$ for dirty [ 8 ]. In 1991, a follow-up study demonstrated reduced infection rates within NNIS System hospitals-a finding due to the implementation of sterile protocols and advancements in surgical technique. SSI rates reported in this study were as follows: $2.1 \%$ for clean, $3.3 \%$ for clean/contaminated, $6.4 \%$ for contaminated, and $7.1 \%$ for dirty cases [9]. This corroborated data from a study by Olson and Lee [10] in 1990 that found reduced rates of infection in

\section{Table 1. ACS-NSOIP surgical wound classifications [11]}

\begin{tabular}{|ll}
\hline Clean & $\begin{array}{l}\text { Uninfected operative wounds without inflammation; respiratory, } \\
\text { alimentary, genital or uninfected urinary tracts are not entered }\end{array}$ \\
Clean/ & $\begin{array}{l}\text { Operative wounds in the respiratory, alimentary, genital or } \\
\text { Contaminated } \\
\text { uninfected urinary tracts are electively entered; without unusual } \\
\text { contamination }\end{array}$ \\
Contaminated & $\begin{array}{l}\text { Open, fresh, accidental wounds, operations with major breaks in } \\
\text { sterile technique or gross spillage from the gastrointestinal tract, } \\
\text { and incisions in which acute, non-purulent inflammation is } \\
\text { encountered }\end{array}$ \\
Dirty & $\begin{array}{l}\text { Old traumatic wounds with retained devitalized tissue or those } \\
\text { that involve existing clinical infection or perforated viscera }\end{array}$
\end{tabular}

ACS-NSQIP, American College of Surgeons-the National Surgical Quality Improvement Program. clean, clean/contaminated, and contaminated procedures compared to rates from 1977.

\section{Surgical quality improvement}

Many institutions and programs are making continued efforts to elevate the quality of patient care. Specifically, the National Healthcare Safety Network (NHSN) integrates patient and healthcare personnel safety surveillance information for the CDC; the Agency for Healthcare Research and Quality (AHRQ) aims to improve the quality, safety, efficiency, and effectiveness of healthcare for Americans; the National Surgical Quality Improvement Program (NSQIP) database prospectively tracks surgical outcomes for the American College of Surgeons (ACS). The AHRQ and NSQIP have brought quality improvement to the forefront of medical discussions.

The NSQIP database is a nationally validated surgical outcomes database that houses 240 preoperative, intraoperative, and postoperative variables from over 250 institutions [11]. It has allowed for multi-institutional, retrospective studies to evaluate surgical outcomes. While previous studies have evaluated surgical site infection rates by wound classification across surgical subspecialties in NSQIP, no studies have utilized this database to look at plastic surgery specifically. Thus far, studies investigating postoperative infections within the field of plastic surgery have been limited to a few types of procedures and/or a single institution. This present study aims to use the ACS-NSQIP database to evaluate surgical site infections by wound classification in plastic surgery cases between 2006 and 2010.

\section{METHODS}

\section{ACS-NSQIP}

Data is extracted from patient medical records, physician office records, and telephone interviews by trained surgical clinical nurse reviewers (SCNRs). All information is subsequently deidentified [11].

\section{Ethical approval}

De-identified patient information is freely available to all institutional members who comply with the ACS-NSQIP Data Use Agreement. The Data Use Agreement implements the protections afforded by the Health Insurance Portability and Accountability Act of 1996 and the ACS-NSQIP Hospital Participation Agreement. This study conforms to the Helsinki Declaration. The ACS-NSQIP and the hospitals participating in the ACSNSQIP are the source of the data used herein; they have not verified and are not responsible for the statistical validity of the data analysis or the conclusions derived by the authors. 


\section{Patient population}

We retrospectively reviewed database participant files from 2006-2010 for all cases with 'plastics' recorded as the primary service. At total of 15,289 plastic surgery cases were identified. No cases within this cohort were without proper wound classification designation, and no patients died on the same day of surgery.

\section{Outcomes}

The outcomes of interest in this study were SSIs, classified as superficial, deep, or organ/space, overall complications, reoperation, and mortality. An SSI was defined in the ACS-NSQIP database, based on CDC criteria, as an infection within 30 days of the primary operation that had purulent drainage with or without laboratory confirmation, organisms isolated from an aseptically obtained culture, and/or a diagnosis by the attending surgeon. Identification of an abscess indicated a deep or organ/ space SSI. Additionally, at least one of the following signs or symptoms must have been present: pain or tenderness, localized swelling, redness, or warmth, an incision deliberately opened by the attending surgeon, or in the case of deep SSI, fever $>38^{\circ} \mathrm{C}$. The depth and location of the infection determined the type of SSI. A superficial SSI involved only the skin or subcutaneous tissues of the incision; a deep SSI involved the muscle and fascial layers; an organ/space SSI involved any part of the anatomy. An infection involving both the superficial and deep tissues was recorded as a deep SSI, as was an organ/space SSI that drained through the incision $[12,13]$.

Overall SSI was defined as $\geq 1$ superficial SSI, deep SSI, or organ/space SSI. Overall complication was defined as having $\geq 1$ of the following ACS-NSQIP postoperative adverse events: superficial SSI, deep SSI, organ/space SSI, wound disruption/ dehiscence, pneumonia, unplanned intubation, pulmonary embolism, failure to wean from ventilator, renal insufficiency, progressive renal failure, urinary tract infection, stroke, coma, peripheral neurologic deficiency, cardiac arrest, myocardial infarction, bleeding requiring a transfusion, deep venous thrombosis (DVT), and sepsis/septic shock. All complication, reoperation, and mortality rates were tracked for 30 days postoperatively.

\section{Risk-adjustment factors}

Patient demographics and medical comorbidities were tracked as potential confounders. Demographic data collected included age, gender, and race. Medical comorbidities included obesity (body mass index $[\mathrm{BMI}] \geq 30$ ), diabetes, dyspnea, ascites, renal disease, chronic obstructive pulmonary disease (COPD), current pneumonia, ventilator dependence, chronic steroid use, bleeding disorders, heart failure, myocardial infarction within 6 months of operation, peripheral vascular disease, disseminated cancer, weight loss of $>10 \%$ body weight within 6 months of operation, current chemotherapy or radiotherapy, neurologic deficit, preoperative transfusion, and preoperative sepsis. Alcohol use, defined as $>2$ drinks per day, and smoking status were tracked as behavioral risk factors. Additionally, operative time, a recognized risk factor for SSI, was included in the risk adjustment.

\section{Statistical analysis}

Patient demographics, risk factors, and postoperative outcomes were compared using chi-square tests for categorical variables and independent $t$-tests for continuous variables, with significance set at $\mathrm{P} \leq 0.05$.

Multivariable logistic regression analysis was conducted to examine the effect of wound classification on 30-day postoperative surgical site infections, overall complications, reoperation, and mortality. Potential confounding risk factors were determined using a bivariate screen that identified variables with significance at $\mathrm{P} \leq 0.20$. All data analyses were performed using SPSS ver. 20.0 (IBM Corp., Armonk, NY, USA).

\section{RESULTS}

A total of 15,289 plastic surgery cases were extracted from the database. No cases were excluded. From this cohort, $81.95 \%$ of the procedures were classified as clean, $7.18 \%$ clean/contaminated, $5.56 \%$ contaminated, and $5.30 \%$ dirty (Table 2). Over three-fourths of the patients were female, and nearly the same proportion was Caucasian. The average age of the group was 48.36 years, with equal distribution between the 40 to 49,50 to 59 , and $\geq 60$ year old age brackets. The most common comorbidity was obesity at $33.61 \%$, followed by diabetes at $8.29 \%$. The two most common preoperative risk factors were hypertension $(27.39 \%)$ and smoking (18.17\%). Four-hundred and six of the total cases (2.66\%) were classified as emergency cases. When comparing patients with and without recorded surgical site infections, there were notable differences in obesity (53.69\% vs. $33.68 \%, \mathrm{P}<0.001)$, diabetes $(15.16 \%$ vs. $8.08 \%, \mathrm{P}<0.001)$ and hypertension ( $37.58 \%$ vs. $27.09 \%, \mathrm{P}<0.001)$. There were also significant differences in the proportion of patients with a history of COPD ( $3.74 \%$ vs. $1.78 \%, \mathrm{P}=0.001)$ and on dialysis $(1.76 \%$ vs. $0.57 \%, \mathrm{P}=0.001)$, although the overall rates were low.

There were 998 cases with a postoperative complication, representing $6.53 \%$ of the total plastic surgery procedures analyzed (Table 3). Nearly one-fifth of dirty procedures incurred a post operative complication, compared to $15.76 \%$ of contaminated cases, $9.20 \%$ of clean/contaminated, and $4.81 \%$ of clean cases 
(Table 3). Surgical site infections (SSIs) made up the majority of these postoperative complications, occurring in 458 cases (3.00\%). Reoperation and mortality rates also increased as the wound classification transitioned from clean to dirty (Table 3 ).

Overall, $1.89 \%$ of plastic surgery patients suffered a superficial SSI, $0.81 \%$ experienced a deep SSI, and $0.35 \%$ had an organ/ space SSI. Superficial SSIs occurred at similar rates across wound

Table 2. Patient demographics and preoperative conditions of 2006-2010 ACS-NSO.|P plastic surgery cases $(n=15,289)$

\begin{tabular}{|lc|}
\hline Characteristic & No. (\%) \\
\hline Gender & \\
Male & $3,351(21.99)$ \\
Female & $11,890(78.01)$ \\
Age category (yr) & \\
$<20$ & $303(1.99)$ \\
$20-29$ & $1,714(11.21)$ \\
$30-39$ & $2,420(15.83)$ \\
$40-49$ & $3,549(23.21)$ \\
$50-69$ & $3,620(23.68)$ \\
$\geq 70$ & $3,683(23.09)$ \\
Ethnicity & \\
White & $11,212(73.33)$ \\
Black & $1,584(10.36)$ \\
Asian & $224(1.47)$ \\
Other & $2,269(14.84)$ \\
Preoperative risk factor/co-morbidity & \\
BMl $\geq 30$ (kg/m²) & $5,139(33.61)$ \\
Smoking & $2,778(18.17)$ \\
Diabetes & $1,267(8.29)$ \\
Alcohol use & $310(2.03)$ \\
COPD & $281(1.84)$ \\
Hypertension & $4,188(27.39)$ \\
Dialysis & $93(0.61)$ \\
Steroid use & $260(1.70)$ \\
Chemo or radiation & $265(1.73)$ \\
No. of emergency cases & $406(2.66)$ \\
Wound classification & \\
Clean & $12,530(81.95)$ \\
Clean/contaminated & $1,098(7.18)$ \\
Contaminated & $850(5.56)$ \\
Dirty & $811(5.30)$ \\
\hline ACS-NSQIP, American College of Surgeons-the National Surgical Quality Improvement \\
Program; BMl, body mass index; COPD, chronic obstructive 0 pulmonary disease. \\
\hline
\end{tabular}

classification cohorts: $1.89 \%$ of clean cases, $1.64 \%$ of clean/contaminated cases, $2.24 \%$ of contaminated cases, and $1.97 \%$ of dirty cases. There were similar rates of occurrence for deep SSIs in the clean and clean/contaminated groups $(0.64 \%)$, while $2.24 \%$ of contaminated cases and $2.22 \%$ of the dirty cases experienced a deep SSI. Organ/space SSI occurred in less than $1 \%$ of each wound classification cohort (Tables 3,4 ). When compared to all other specialties, plastic surgery had lower rates of surgical site infections in clean/contaminated, contaminated, and dirty cases (Table 4).

Adjusted odds ratios (OR) and 95\% confidence intervals (CI) for superficial, deep, and organ/space SSIs are listed in Table 5. With clean operations as a reference group, clean/contaminated, contaminated, and dirty cases had a statistically similar risk of a superficial SSI. The odds ratios for deep SSIs were 0.84 (95\% CI, 0.38-1.87; $\mathrm{P}=0.666$ ), 2.81 (95\% CI, 1.43-5.50; $\mathrm{P}=0.003$ ), and $2.74(95 \% \mathrm{CI}, 1.36-5.52 ; \mathrm{P}=0.005)$ for clean/contaminated, contaminated, and dirty cases, respectively. Wound classification did not appear to be a significant predictor of an organ/space SSI; the odds ratios for an organ/space SSI were 1.48 (95\% CI, 0.61-3.60; $\mathrm{P}=0.399), 0.81$ (95\% CI, 0.23-2.82, $\mathrm{P}=0.731$ ), and 1.73 (95\% CI, 0.60-5.05; $\mathrm{P}=0.322$ ) for clean/contaminated, contaminated, and dirty procedures, respectively.

Regression analysis was also conducted on our remaining outcomes of interest-overall complications, reoperation, and overall mortality (Table 6). Clean/contaminated procedures were comparable to clean cases in their risk of reoperation and mortality. However, clean/contaminated procedures increased

Table 4. Comparison of overall postoperative SSIs reported in ACS-NSO.|P stratified by wound classification [13]

\begin{tabular}{|lcccc|}
\hline Population & Clean & $\begin{array}{c}\text { Clean/Con- } \\
\text { taminated }\end{array}$ & $\begin{array}{c}\text { Contami- } \\
\text { nated }\end{array}$ & Dirty \\
\hline All specialties & $2.58 \%$ & $6.67 \%$ & $8.61 \%$ & $11.80 \%$ \\
Plastic surgery & $2.75 \%$ & $2.82 \%$ & $4.94 \%$ & $5.06 \%$ \\
\hline ACS & & &
\end{tabular}

ACS-NSQIP, American College of Surgeons-the National Surgical Quality Improvement Program; SSls, surgical site infections.

a)Denotes significant value, $\mathrm{P}<0.05$.

\section{Table 3. Rates of overall complications, surgical site infections, reoperation, and mortality by wound classification}

\begin{tabular}{|c|c|c|c|c|}
\hline Postoperative outcomes & Clean $(n=12,530)$ & Clean/Contaminated $(n=1,098)$ & Contaminated $(n=850)$ & Dirty $(n=811)$ \\
\hline Overall complications & $603(4.81)$ & $101(9.06)$ & $134(15.76)$ & $160(19.73)$ \\
\hline Overall surgical site infection & $344(2.75)$ & $31(2.82)$ & $42(4.94)$ & $41(5.06)$ \\
\hline Superficial SSI & $237(1.89)$ & $18(1.64)$ & $19(2.24)$ & $16(1.97)$ \\
\hline Deep SSI & $80(0.64)$ & $7(0.64)$ & $19(2.24)$ & $18(2.22)$ \\
\hline Organ/Space SSI & $34(0.27)$ & $7(0.64)$ & $4(0.47)$ & $8(0.99)$ \\
\hline Reoperation & $404(3.23)$ & $68(6.19)$ & $100(11.76)$ & $154(18.99)$ \\
\hline Mortality & $10(0.08)$ & $2(0.18)$ & $12(1.41)$ & $28(3.45)$ \\
\hline
\end{tabular}


Table 5. Risk-adjusted regression analysis: surgical site infections stratified by wound classification

\begin{tabular}{|c|c|c|c|c|c|c|}
\hline \multirow{2}{*}{ Wound classification } & \multicolumn{2}{|c|}{ Superficial SSI ${ }^{a)}$} & \multicolumn{2}{|c|}{ Deep SSI ${ }^{b)}$} & \multicolumn{2}{|c|}{ Organ/Space SSI ${ }^{c}$} \\
\hline & OR & $95 \% \mathrm{Cl}$ & $\mathrm{OR}$ & $95 \% \mathrm{Cl}$ & OR & $95 \% \mathrm{Cl}$ \\
\hline Clean & \multicolumn{2}{|c|}{ Reference } & \multicolumn{2}{|c|}{ Reference } & \multicolumn{2}{|c|}{ Reference } \\
\hline Clean/Contaminated & 0.75 & $0.46-1.24$ & 0.84 & $0.38-1.87$ & 1.48 & $0.61-3.60$ \\
\hline Contaminated & 0.93 & $0.52-1.67$ & $2.81^{d)}$ & $1.43-5.50$ & 0.81 & $0.23-2.82$ \\
\hline Dirty & 0.77 & $0.41-1.45$ & $2.74^{d)}$ & $1.36-5.52$ & 1.73 & $0.60-5.05$ \\
\hline
\end{tabular}

Table 6. Risk-adjusted regression analysis: overall complication, reoperation, and mortality stratified by wound classification

\begin{tabular}{|c|c|c|c|c|c|c|c|c|c|}
\hline \multirow{2}{*}{ Wound classification } & \multicolumn{3}{|c|}{ Overall complication $^{\text {a) }}$} & \multicolumn{3}{|c|}{ Reoperation $^{\text {b) }}$} & \multicolumn{3}{|c|}{ Mortalityc) } \\
\hline & OR & \multicolumn{2}{|c|}{$95 \% \mathrm{Cl}$} & OR & \multicolumn{2}{|c|}{$95 \% \mathrm{Cl}$} & OR & \multicolumn{2}{|c|}{$95 \% \mathrm{Cl}$} \\
\hline Clean & \multicolumn{3}{|c|}{ Reference } & \multicolumn{3}{|c|}{ Reference } & \multicolumn{3}{|c|}{ Reference } \\
\hline Clean/Contaminated & $1.33^{\text {d) }}$ & 1.03 & 1.71 & 1.23 & 0.93 & 1.65 & 1.34 & 0.28 & 6.50 \\
\hline Contaminated & $1.90^{d)}$ & 1.44 & 2.52 & $1.69^{d)}$ & 1.25 & 2.30 & $6.35^{d)}$ & 2.23 & 18.06 \\
\hline Dirty & $2.28^{\mathrm{d})}$ & 1.72 & 3.01 & $2.68^{d)}$ & 2.01 & 3.59 & $10.55^{d)}$ & 3.90 & 28.54 \\
\hline
\end{tabular}

$\mathrm{OR}$, odds ratio; $\mathrm{Cl}$, confidence interval.

${ }^{a)}$ Additional variables included in regression for overall complications include male gender, race, age, obesity, diabetes, smoking, dyspnea, hypertension, dialysis, disseminated cancer, recent wound infection, chronic steroid use, known bleeding disorder, prior chemotherapy, prior radiotherapy, prior sepsis, prior operation, operative time (hr), and emergency case; ")Variables included in regression analysis for reoperation were the same as the variables for overall complication analysis except that prior chemotherapy did not reach significance on bivariate screening and was not included; ' ${ }^{c}$ Variables incorporated into regression analysis for mortality included male gender, age, diabetes, dyspnea, hypertension, dialysis, disseminated cancer, recent wound infection, steroid use, known bleeding disorder, prior chemotherapy, prior sepsis, prior operation, operative time (hr), and emergency case; 'Denotes significant value, $P<0.05$.

the risk of overall complications by $33 \%$ (adjusted OR, 1.33; 95\% CI, 1.03-1.71; $\mathrm{P}<0.001)$. Contaminated cases were associated with adjusted ORs of 1.90 for overall complications (95\% CI, 1.44-2.52; $\mathrm{P}<0.001$ ), 1.69 for reoperation (95\% CI, 1.252.30; $\mathrm{P}=0.001$ ), and 6.35 for 30-day mortality ( $95 \% \mathrm{CI}$, 2.2318.06; $\mathrm{P}=0.001$ ). Dirty cases had a 2.28 greater odds of having a postoperative complication ( $95 \% \mathrm{CI}, 1.72-3.01 ; \mathrm{P}<0.001)$, a 2.68 greater odds of reoperation (95\% CI, 2.01-3.59; $\mathrm{P}<0.001$ ), and a 10.55 greater odds of 30 -day mortality (95\% CI, 3.90-28.54; $\mathrm{P}<0.001)$ when compared to clean cases

\section{DISCUSSION}

This study is the first to investigate surgical site infections in plastic surgery by wound classification using a nationally validated surgical outcomes database. Overall, surgical site infection rates for plastic surgery were lower in non-clean, i.e. clean/contaminated, contaminated, and dirty, cases than the most recently reported rates for all surgical specialties represented by ACS [14]. Additionally, our infection rates by wound class were universally lower than rates previously reported in a prospective study on plastic surgery cases [15]. Such findings may serve as a benchmark for future outcomes studies. Over $80 \%$ of plastic surgery procedures were classified as clean, compared to approximately $50 \%$ of procedures across all ACS specialties [14]. These numbers reflect a selection bias in plastic surgery with respect to surgical site infections and a tendency of plastic surgery operations to focus on the soft tissues.

Plastic surgery is acknowledged as a diverse specialty, with procedures addressing a variety of anatomic locations and representing a range of technical complexity. A recent literature review found the rate of SSIs in plastic surgery to vary greatly with the type of surgery performed: $0.001 \%$ to $7 \%$ in breast augmentation, 1.1 to $22 \%$ in breast reductions, $0.2 \%$ to $32.6 \%$ in abdominoplasty, and only $0 \%$ to $0.3 \%$ in rhytidectomy and less than $0.1 \%$ in browlift [16]. Three of the four most common clean procedures captured in our study were focused on the breast, with mammaplasty being the most prevalent (19.80\%).

In contrast, over $36 \%$ of dirty cases were pressure sore and flap procedures. Our total SSI rate of 3.00\% (458 of 15,289 patients) is consistent with published reports in the plastic surgery literature [16-18]. As a finding from a multi-center database, our data regarding surgical infection rates may supersede the implied biases of individual surgical series based on only a few surgeons or institutions, as seen in previous studies. A prospective study conducted by Andenaes et al. [15] found that wound infection 
rates escalated from $10.2 \%$ in clean plastic surgery cases to $37.5 \%$ in dirty plastic surgery cases. Our overall SSI rates were much lower-ranging from $2.74 \%$ in clean procedures to $5.06 \%$ in dirty operations. The discrepancy in infection rates is most likely attributable to the fact that our study utilized a more stringent definition of infection, based on CDC criteria; the study by Andenaes et al. [15] employed a Wound Infection Score (WIS) system that included a single presentation of "edema," "redness," or "increased pain" in their definition of wound infection. Additionally, a learning curve may exist, with plastic surgeons being more selective when choosing patients for specific operations at the present time compared to the years captured by the Andenaes study.

Our results showed no observable incremental increase in infection rates when progressing from clean to dirty cases. While there does appear to be a slight correlation between wound classification and overall SSI, the relationship breaks down when stratified by type of infection. The highest rate of organ/space surgical site infections was associated with dirty procedures; however, the highest rates of superficial and deep SSIs occurred in contaminated operations. Our risk-adjusted multivariable regression model revealed that wound classification was not a significant predictor for two out of the three types of surgical site infections, namely superficial SSIs and organ/space SSIs. Contaminated and dirty wound categories were significant independent predictors of a deep SSI, associated with a nearly threefold increased risk of a deep SSI. These findings are in contrast to the study by Ortega et al. [14], which analyzed infection rates by wound class across all surgical specialties captured in NSQIP. They demonstrated an increased rate of infection when advancing from clean to dirty procedures and found wound classification to be a significant predictor of superficial, deep, and organ/ space surgical site infections by multivariate analyses.

The reasoning for the lower infection rates in non-clean plastic surgery cases compared to similar cases in the Ortega study is multifaceted. Patient selection is a presumed contributor, with plastic surgeons operating on individuals who may be at a baseline lower risk for infections. Additionally, the majority of surgical site infections were classified as superficial and thus involved only skin or subcutaneous tissue. Plastic surgeons are trained in procedures involving soft tissues and therefore might have invested more effort into techniques such as debridement of contaminated or devitalized tissues, dead space reduction, and meticulous layered closure to reduce the likelihood of complications in the superficial and deep tissue layers. The discrepancies in infection rates by wound class could also be due in part to the differences in the procedure characteristics that define the nonclean classifications. In the majority of non-clean procedures in the Ortega study, the alimentary tract was entered. Plastic surgeons only occasionally enter the alimentary or respiratory tracts, so the majority of their non-clean procedures are likely to be those associated with open wounds or trauma. Moreover, these non-clean procedures may involve surgical debridement or preparation of the surgical bed as the first step of the reconstructive operation. Thus, the operation is a treatment for the contaminated wound rather than the contaminated wound being a consequence of the procedure.

Although statistical analysis did not support wound classification as a predictor of most types of surgical site infections, it was proven to be significant in predicting overall complications. In acknowledgement of the baseline discrepancies in patients with and without recorded surgical site infections, variables that reached significance on bivariate screening were included in the regression analysis. Clean/contaminated cases showed a 1.33 increased odds of having a postoperative complication (95\% CI, 1.03-1.71; P = 0.027), contaminated cases held a 1.9 increased odds of having a postoperative complication (95\% CI, 1.44-2.52; $\mathrm{P}<0.001)$, and dirty cases had a 2.28 increased odds of having a complication (95\% CI, 1.72-3.01; P < 0.001). It has been demonstrated that contaminated wounds containing greater than 105 bacteria per gram of tissue are not effectively treated by skin grafting, primary closure, or random cutaneous flaps. It has further been shown that in heavily contaminated wounds containing greater than 106 bacteria per gram of tissue, musculocutaneous flaps will dehisce [19]. Such flap and graft failures may have been tracked as complications other than surgical site infections by NSQIP.

Wound classification was also a significant predictor of reoperation. Contaminated operations had a $69 \%$ increased risk for reoperation, and dirty procedures had over a $100 \%$ increased risk for reoperation. Additionally, contaminated and dirty cases were significant predictors for mortality, with contaminated procedures having an OR of 6.35 and dirty procedures having an OR of 10.55. While NSQIP does not track the reason for reoperation or cause of death, these results may reflect the possibility of increased microbial contamination contributing to instances that lead to reoperation or death, such as wound breakdown and sepsis. Moreover, it may be that patients in a wound classification with a higher bacterial burden have preoperative characteristics and comorbidities that place them at increased risk for such adverse events.

The wound classification system was created to have universal utility in identifying procedures with an inherently higher risk of postoperative infection. However, the large majority of plastic surgery cases are clean cases, and the definitions of the other classifications as they pertain to plastic surgery are less clear. One 
single institutional study of wound classification found 19\% of their cases to be misclassified [20]. When the ACS-NSQIP data was narrowed to study plastic surgery alone, wound classification lost much if its significance as a predictor for surgical site infection. The classification scheme does not consider basic plastic surgical principles such as the inherent vascularity of the surgical site or the use of prosthetic material. A modified wound classification system may be necessary to strengthen its applicability to specific fields and help further standardize preoperative antibiotic regimens and surgical advancements.

Despite suggestions that the nearly 50-year-old classification system should be revised, no such modifications have been adopted [21,22]. However, Culver et al. [9] did devise the NNIS SSI risk index, which did not address the wound classification scheme itself, but combined it with other known risk factors to improve predictive value. The NNIS SSI risk index assigned point values to the following three criteria: American Society of Anesthesiologists score of 3 or greater, wound classification of either contaminated or dirty, and procedure-specific excessive operative time. The risk index proved to be a better predictor of SSIs than wound classification alone. Optimally, however, a wound classification system could serve as a tool for risk-adjustment specific to the wound characteristics, e.g., size, location, depth, approach, and level of contamination, and would be independent of patient and other operative variables. We therefore suggest that any future modification of the wound classification system consider such focused variables as anticipated incision size, detailed anatomic locations, and surgical approach (laparoscopic versus open) in addition to the level of contamination to better predict infections.

Despite the statistical power of this multi-institutional database, there are limitations to this study. First, the database tracks only 30-day postoperative outcomes and cannot properly capture a second stage reconstructive procedure. A focus on 30-day outcomes eliminates the possibility of tracking implant infections defined up to 1 year after the initial operation. Furthermore, data is recorded once every eight days, which may have impacted the proportion of plastic surgery operations captured in our analysis. Additionally, the database has limitations regarding variables germane to plastic and reconstructive surgery. In particular, the rate of seroma, a common postoperative complication, is absent, which therefore reduces the attributable complication rate. Particularly pertinent to this study is the lack of preoperative and postoperative care variables, specifically regarding the use of antibiotics and drains. Although nothing specific is captured, it is assumed that every institution will have some form of antibiotic prophylaxis in place. As the database is revised on an annual basis, it is our hope that the aforementioned untracked variables are incorporated to further enhance future studies.

\section{REFERENCES}

1. Klevens RM, Edwards JR, Richards CL Jr, et al. Estimating health care-associated infections and deaths in U.S. hospitals, 2002. Public Health Rep 2007;122:160-6.

2. Thompson KM, Oldenburg WA, Deschamps C, et al. Chasing zero: the drive to eliminate surgical site infections. Ann Surg 2011;254:430-6.

3. Boyce JM, Potter-Bynoe G, Dziobek L. Hospital reimbursement patterns among patients with surgical wound infections following open heart surgery. Infect Control Hosp Epidemiol 1990;11:89-93.

4. Olsen MA, Chu-Ongsakul S, Brandt KE, et al. Hospital-associated costs due to surgical site infection after breast surgery. Arch Surg 2008; 143:53-60.

5. Poulsen KB, Bremmelgaard A, Sorensen AI, et al. Estimated costs of postoperative wound infections. A case-control study of marginal hospital and social security costs. Epidemiol Infect 1994;113:283-95.

6. Kirkland KB, Briggs JP, Trivette SL, et al. The impact of surgical-site infections in the 1990s: attributable mortality, excess length of hospitalization, and extra costs. Infect Control Hosp Epidemiol 1999;20:725-30.

7. Hart D, Postlethwait RW, Brown IW Jr, et al. Postoperative wound infections: a further report on ultraviolet irradiation with comments on the recent (1964) national research council cooperative study report. Ann Surg 1968;167:728-43.

8. Garner JS. CDC guideline for prevention of surgical wound infections, 1985. Supersedes guideline for prevention of surgical wound infections published in 1982. (Originally published in November 1985). Revised. Infect Control 1986;7: 193-200.

9. Culver DH, Horan TC, Gaynes RP, et al. Surgical wound infection rates by wound class, operative procedure, and patient risk index. National Nosocomial Infections Surveillance System. Am J Med 1991;91:152S-7S.

10. Olson MM, Lee JT Jr. Continuous, 10-year wound infection surveillance. Results, advantages, and unanswered questions. Arch Surg 1990;125:794-803.

11. American College of Surgeons. ACS data collection, analysis, and reporting [Internet]. Chicago, IL: American College of Surgeons; c2013 [cited 2012 Aug 31]. Available from: http://site.acsnsqip.org/programspecifics/data-collectionanalysis-and-reporting/.

12. Horan TC, Gaynes RP, Martone WJ, et al. CDC definitions of nosocomial surgical site infections, 1992: a modification 
of CDC definitions of surgical wound infections. Infect Control Hosp Epidemiol 1992;13:606-8.

13. American College of Surgeons. ACS NSQIP: user guide for the 2009 participant use data file [Internet]. Chicago, IL: American College of Surgeons; c2010 [cited 2012 Oct 31]. Available from: http://site.acsnsqip.org/wp-content/uploads/2012/03/ACS_NSQIP_Participant_User_Data_ File_User_Guide.pdf.

14. Ortega G, Rhee DS, Papandria DJ, et al. An evaluation of surgical site infections by wound classification system using the ACS-NSQIP.J Surg Res 2012;174:33-8.

15. Andenaes K, Amland PF, Lingaas E, et al. A prospective, randomized surveillance study of postoperative wound infections after plastic surgery: a study of incidence and surveillance methods. Plast Reconstr Surg 1995;96:948-56.

16. Gravante G, Caruso R, Araco A, et al. Infections after plastic procedures: incidences, etiologies, risk factors, and antibi- otic prophylaxis. Aesthetic Plast Surg 2008;32:243-51.

17. Drapeau CM, D’Aniello C, Brafa A, et al. Surgical site infections in plastic surgery: an Italian multicenter study. J Surg Res 2007;143:393-7.

18. Sylaidis P, Wood S, Murray DS. Postoperative infection following clean facial surgery. Ann Plast Surg 1997;39:342-6.

19. Murphy RC, Robson MC, Heggers JP, et al. The effect of microbial contamination on musculocutaneous and random flaps. J Surg Res 1986;41:75-80.

20. Devaney L, Rowell KS. Improving surgical wound classification: why it matters. AORN J 2004;80:208-9.

21. Eisenberg D. Surgical site infections: time to modify the wound classification system? J Surg Res 2012;175:54-5.

22. Nichols RL. Classification of the surgical wound: a time for reassessment and simplification. Infect Control Hosp Epidemiol 1993;14:253-4. 